\title{
Combinatorial signaling codes for the progressive determination of cell fates in the Drosophila embryonic mesoderm
}

\author{
Ana Carmena, ${ }^{1,3}$ Stephen Gisselbrecht, ${ }^{2,3}$ Jacob Harrison, ${ }^{2}$ Fernando Jiménez, ${ }^{1}$ \\ and Alan M. Michelson ${ }^{2,4}$ \\ ${ }^{1}$ Centro de Biologia Molecular 'Severo Ochoa' (CSIC-UAM), Universidad Autónoma, 28049 Madrid, Spain; ${ }^{2}$ Division of \\ Genetics, Department of Medicine, Brigham and Women's Hospital, Harvard Medical School and Howard Hughes Medical \\ Institute, Boston, Massachusetts 02115 USA
}

\begin{abstract}
Mesodermal progenitors arise in the Drosophila embryo from discrete clusters of lethal of scute (l'sc)-expressing cells. Using both genetic loss-of-function and targeted ectopic expression approaches, we demonstrate here that individual progenitors are specified by the sequential deployment of unique combinations of intercellular signals. Initially, the intersection between the Wingless $(\mathrm{Wg})$ and Decapentaplegic (Dpp) expression domains demarcate an ectodermal prepattern that is imprinted on the adjacent mesoderm in the form of a L'sc precluster. All mesodermal cells within this precluster are competent to respond to a subsequent instructive signal mediated by two receptor tyrosine kinases (RTKs), the Drosophila epidermal growth factor receptor (DER) and the Heartless (Htl) fibroblast growth factor receptor. By monitoring the expression of the diphosphorylated form of mitogen-associated protein kinase (MAPK), we found that these RTKs are activated in small clusters of cells within the original competence domain. Each cluster represents an equivalence group because all members initially resemble progenitors in their expression of both L'sc and mesodermal identity genes. Thus, localized RTK activity induces the formation of mesodermal equivalence groups. The RTKs remain active in the single progenitor that emerges from each cluster under the subsequent inhibitory influence of the neurogenic genes. Moreover, DER and Htl are differentially involved in the specification of particular progenitors. We conclude that distinct cellular identity codes are generated by the combinatorial activities of Wg, Dpp, EGF, and FGF signals in the progressive determination of embryonic mesodermal cells.
\end{abstract}

[Key Words: Wingless; Decapentaplegic; receptor tyrosine kinase; equivalence group; myogenesis; cardiogenesis]

Received July 24, 1998; revised version accepted October 29, 1998.

During animal development, a wide diversity of cellular identities must be specified within initially undifferentiated fields of cells. One solution to this problem is for a hierarchy of regulators to promote the progressive determination of cells, essentially carving out from the original field domains with increasingly restricted developmental potential. In such a mechanism, spatially localized factors first delineate a prepattern in which all cells are equally competent to adopt a particular identity (Stern 1954; Greenwald and Rubin 1992). The expression of additional regulatory molecules in cellular subsets within the prepatterned territory further limits the responses afforded particular cells. Precise refinement of the final pattern can be dictated by direct inhibitory interactions among neighboring cells /Greenwald and Ru-

\footnotetext{
${ }^{3}$ These authors contributed equally to this work.

${ }^{4}$ Corresponding author.

E-MAIL michelson@rascal.med.harvard.edu; FAX (617) 738-5575.
}

bin 1992; Simpson 1997). Although many details are known about the later pattern forming steps in a number of developmental systems, relatively little information is available for how early prepatterns are established (Greenwald and Rubin 1992; Kornfeld 1997; Simpson 1997; Vervoort et al. 1997).

The Drosophila embryonic mesoderm provides an ideal system in which to investigate prepattern and pattern formation. The mesoderm arises from the ventral most cells of the blastoderm embryo under the influence of the zygotic genes, twist (twi) and snail (sna). Cells expressing these genes invaginate through the ventral furrow at gastrulation. Subsequently, the internalized mesodermal cells migrate dorsolaterally to form a uniform sheet beneath the ectoderm (Bate 1993; Leptin 1995), a process that is controlled by a fibroblast growth factor (FGF) receptor encoded by heartless (htl; Beiman et al. 1996; Gisselbrecht et al. 1996; Shishido et al. 1997; Michelson et al. 1998). 
Initially, mesodermal cells have relatively unrestricted developmental potentials (Beer et al. 1987). The later allocation of fates is controlled in part by segmentation genes (Azpiazu et al. 1996; Riechmann et al. 1997, 1998). One manifestation of mesodermal segmentation is the generation of alternating domains of high and low Twi expression along the anteroposterior axis, a process that is essential to proper muscle differentiation (Dunin Borkowski et al. 1995; Baylies and Bate 1996). In addition, once definitive positions are established by cell migration, specific mesodermal derivatives are induced by the adjacent ectoderm (Baker and Schubiger 1995). For example, visceral, cardiac, and dorsal somatic muscle fates are induced by Decapentaplegic (Dpp), a transforming growth factor $\beta$-family member that is secreted by the dorsal ectoderm (Staehling-Hampton et al. 1994; Frasch 1995). Wingless (Wg), an ectodermally derived Wnt family member, stimulates the formation of a large subset of somatic muscles and cardiac cells (Bate and Rushton 1993; Baylies et al. 1995; Lawrence et al. 1995; Wu et al. 1995; Park et al. 1996; Ranganayakulu et al. 1996). Autonomous activity of both the Htl FGF receptor and the Drosophila EGF receptor (DER) also contributes to the commitment of various mesodermal cells (Lüer et al. 1997; Buff et al. 1998; Michelson et al. 1998).

Each somatic myofiber derives from a specific mononucleated cell that possesses the information for its unique identity (Bate 1990, 1993). These so-called founder cells seed the formation of individual muscles by fusing with neighboring undifferentiated myoblasts (Rushton et al. 1995). Founders form from the asymmetric division of progenitors, which in turn, are derived from clusters of cells that express the proneural gene, lethal of scute (1'sc; Carmena et al. 1995; Ruiz Gomez and Bate 1997; Carmena et al. 1998). All cells composing a given mesodermal L'sc cluster are initially equipotent. However, only a single progenitor emerges from each cluster through the inhibitory action of the neurogenic genes (Corbin et al. 1991; Bate et al. 1993; Carmena et al. 1995; Baker and Schubiger 1996).

Some mesodermal progenitors require multiple signals for their formation, although it is unclear how these disparate inductive events are coordinated. We have been investigating this question for two subsets of dorsal mesodermal cells that express the pair-rule gene, evenskipped (eve; Frasch et al. 1987). One Eve-positive progenitor gives rise to a pair of pericardial cells, and at least one dorsal somatic muscle forms from a second Eve progenitor (Buff et al. 1998; Carmena et al. 1998). We now report that the two L'sc clusters from which these progenitors arise are prefigured by a broader domain of L'sc expression that is dependent on the combined activities of Wg and Dpp. The corresponding equivalence groups are formed within this prepatterned mesodermal region via localized activation of the Ras1 pathway by two receptor tyrosine kinases (RTKs), Htl, and DER. Whereas $\mathrm{Htl}$ is required for the Eve cardiac equivalence group, both DER and Htl are involved in the Eve muscle cell cluster. These findings demonstrate how positional information initially establishes a mesodermal prepattern, and establish that individual progenitors are progressively determined by unique combinations of intercellular signals.

\section{Results}

A mesodermal prepattern is delineated by the intersecting domains of Wg and Dpp expression

Prior studies demonstrated the existence of small groups of L'sc-expressing mesodermal cells from which the progenitors of the heart and somatic muscles are derived in the Drosophila embryo (Carmena et al. 1995). A more detailed analysis of mesodermal L'sc expression revealed that this proneural gene is initially expressed in two broad patches that prefigure the appearance of the smaller cell clusters. We refer to these patches as L'sc preclusters. One precluster, preC1, is found in the ventral mesoderm, and the other, preC2, is localized to the dorsal mesoderm (Fig. 1A; and data not shown). PreC2 encompasses the territory in which dorsal L'sc clusters C2 and C14-C17 subsequently develop (Carmena et al. 1995; see below).

Wg and Dpp are known to be required for the formation of several mesodermal derivatives (Bate and Rushton 1993; Staehling-Hampton et al. 1994; Baylies et al. 1995; Frasch 1995; Lawrence et al. 1995; Wu et al. 1995; Park et al. 1996; Ranganayakulu et al. 1996). We therefore investigated whether the spatial and temporal patterns of Wg and Dpp expression correlate with the early appearance of L'sc in the underlying mesoderm. During early stage $10, d p p$ transcripts are uniformly found in a dorsal ectodermal band that spans several cell diameters (Ray et al. 1991). Shortly thereafter, $d p p$ RNA is concentrated into patches with alternating high and low expression levels (Fig 1B). At this stage, L'sc is not yet present in the mesoderm, although expression in the nervous system can be detected just ventral to the low $d p p$ domain. By late stage 10, L'sc preC2 appears in the mesoderm beneath the region in which $d p p$ was expressed previously at the highest level (Fig. 1A,C). Although appropriate reagents are not available for the detection of Dpp protein, we can at least conclude that the original dorsoventral boundaries of $d p p$ RNA expression overlap with those of preC2. Similarly, ectodermal Wg expression along the anteroposterior axis anticipates that of $\mathrm{L}^{\prime} \mathrm{sc}$ in the mesoderm (Fig.1D). Once it does appear, preC2 is completely encompassed by the Wg stripe (Fig. 1E). Thus, the intersecting Wg and Dpp domains delineate the anteroposterior and dorsoventral boundaries of a mesodermal L'sc prepattern.

Eve-expressing somatic muscle and cardiac progenitors derive from L'sc precluster 2

To understand the mechanisms responsible for progenitor specification, we have focused on the two dorsal mesodermal derivatives that express Eve. Each embryonic hemisegment contains a pair of Eve-expressing pericar- 
Carmena et al.

Figure 1. Temporal and spatial changes in L'sc and Eve expression in the mesoderm of wild-type embryos. (A) Late-stage 10 embryo stained for expression of L'sc protein. L'sc is expressed in progenitors of the nervous system (NS) and in segmentally repeated patches of dorsal mesodermal cells referred to as precluster 2 (preC2). $(B, C)$ Mid- and late-stage 10 embryos, respectively, stained for expression of L'sc protein (brown) and $d p p$ mRNA (blue). $d p p$ transcripts are intially found in a broad band of ectodermal cells that is modulated into domains of high $\left({ }^{*}\right)$ and low (arrowhead) expression levels. PreC2 forms directly beneath the high $d p p$ domain. The width of the original dorsal $d p p$ stripe spans the entire region that is eventually occupied by preC2. $(D, E)$ Mid- and late-stage 10 embryos, respectively, stained for expression of L'sc (green) and Wg (red). Wg expression precedes L'sc in the mesoderm. When it does appear, preC2 spans the entire $\mathrm{Wg}$ stripe. The asterisks and arrowheads in $B-E$ indicate corresponding positions in the different embryos. $(F-N)$ Progression of L'sc (green) and Eve (red) expression during stages 10 and 11 . The rectangle in $\mathrm{F}$ encompasses the area that is enlarged in $G-N$. (dsm, lsm, vsm) Dorsal, lateral, and ventral somatic mesoderm, respectively. L'sc restricts from preC2 to cluster $2(\mathrm{C} 2$ in $\mathrm{G})$ at late-stage 10, and then Eve is activated in all C2 cells $(H)$. L'sc and Eve restrict to a single progenitor (P2 in $I$ and $J)$, and L'sc initiates in three additional dorsal clusters, C14-C16 $(K ; \mathrm{P} 2$ is out of the plane of focus in this panel). C15, as well as parts of C14 and C16, encompass the territory occupied previously by C2. C15 also expresses Eve, which becomes restricted along with L'sc to the P15 progenitor; additional progenitors, P14, P16, and P17, express only L'sc. P2 and P15 divide to form two sibling founder cells each (F2 and F15). $(A-N)$ Anterior is to the left and dorsal is up.

dial cells (EPCs) and a single Eve-positive dorsal somatic muscle, DA1 (Frasch et al. 1987). The two EPCs derive from a common progenitor, P2, whereas a second progenitor, P15, gives rise to muscle DA1 (Buff et al. 1998; Carmena et al. 1998). P2 develops from L'sc cluster 2 (C2), which initially expresses only L'sc and comprises the dorsalmost cells of preC2 (Fig. 1G). Eve then becomes coexpressed with L'sc in all C2 cells (Fig. 1H). Both L'sc and Eve progressively restrict to a single C2 cell, the $\mathrm{P} 2$ progenitor (Fig. 1I,J). By this time, another set of L'sc clusters, C14-C16, appears in the dorsal mesoderm adjacent to and overlapping with the region that was occupied previously by C2 (Fig. 1K). C15 also expresses Eve and gives rise to the P15 progenitor (Fig. $1 \mathrm{~L}, \mathrm{M})$. Each Eve progenitor divides to form two founder cells (Fig. 1M,N; F2 and F15), but only one founder of each sibling pair retains Eve expression (data not shown). The persistently Eve-positive F2 divides again to form two EPCs per hemisegment, whereas the F15 in which Eve is maintained seeds the formation of muscle DA1 (Buff et al. 1998; Carmena et al. 1998). Because all cells in a given L'sc cluster initially express identity genes such

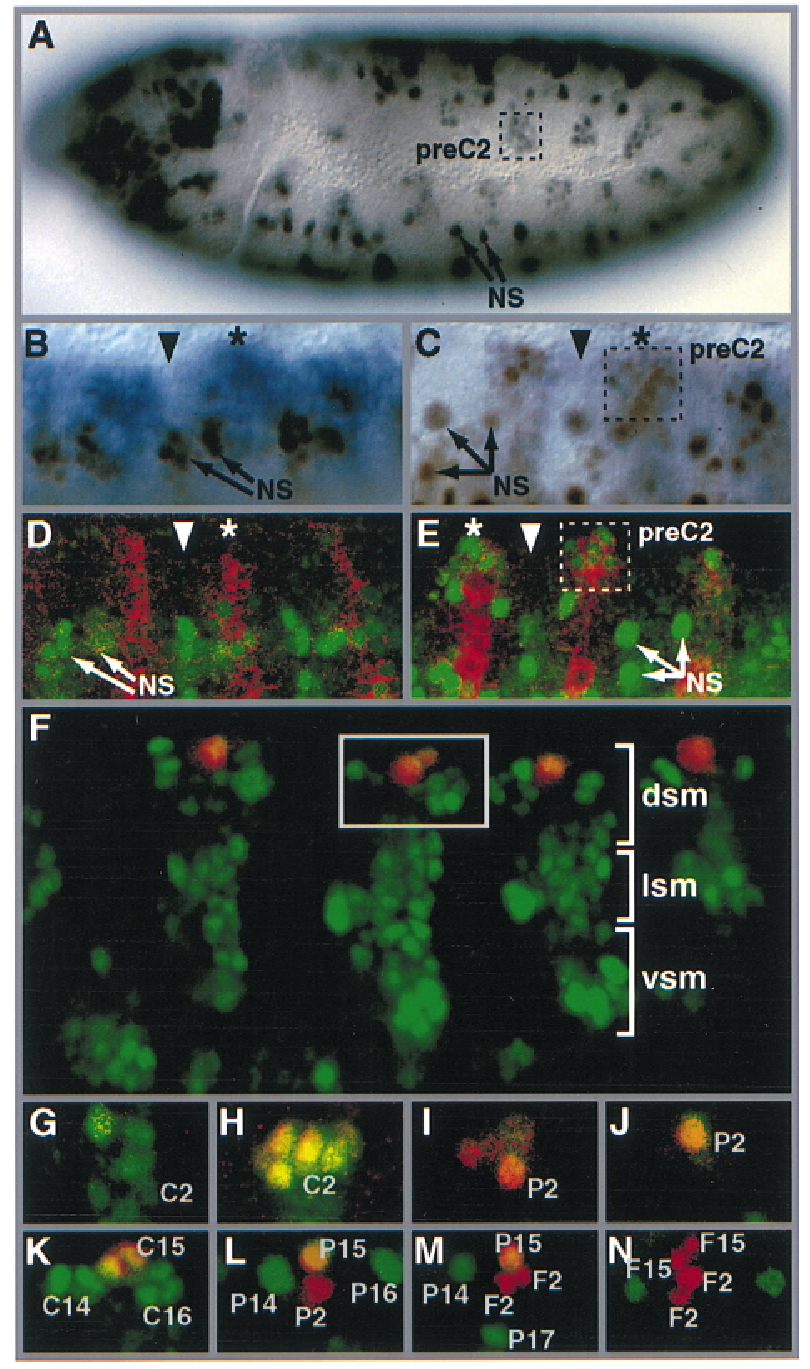

as Eve (Fig. $1 \mathrm{H}, \mathrm{L})$, and identity gene expression persists in the entire cluster in neurogenic mutants (Carmena et al. 1995), such clusters can be considered mesodermal equivalence groups (Greenwald and Rubin 1992).

\section{The mesodermal L'sc prepattern is dependent on $\mathrm{Wg}$ and $D p p$}

Wg and Dpp are both required for the formation of EPCs and the muscle DA1 precursor (Frasch 1995; Lawrence et al. 1995; Wu et al. 1995; Park et al. 1996). Given that the intersecting $\mathrm{Wg}$ and Dpp expression domains precisely correlate with the boundaries of preC2, we investigated whether these two signals play a role in mesodermal L'sc expression. The presence of preC2 is very transient; soon after its appearance, L'sc becomes restricted to the subset of preC2 cells that comprise C2 (Figs. 1G and 2A,B). In wg mutant embryos, neither preC2 nor $\mathrm{C} 2$ develops (Fig. 2C). It was not possible to examine an analogous requirement for Dpp in preC2 or $\mathrm{C} 2$ formation because the shift in dorsoventral fates associated with complete loss of $d p p$ function causes a profound derangement of 


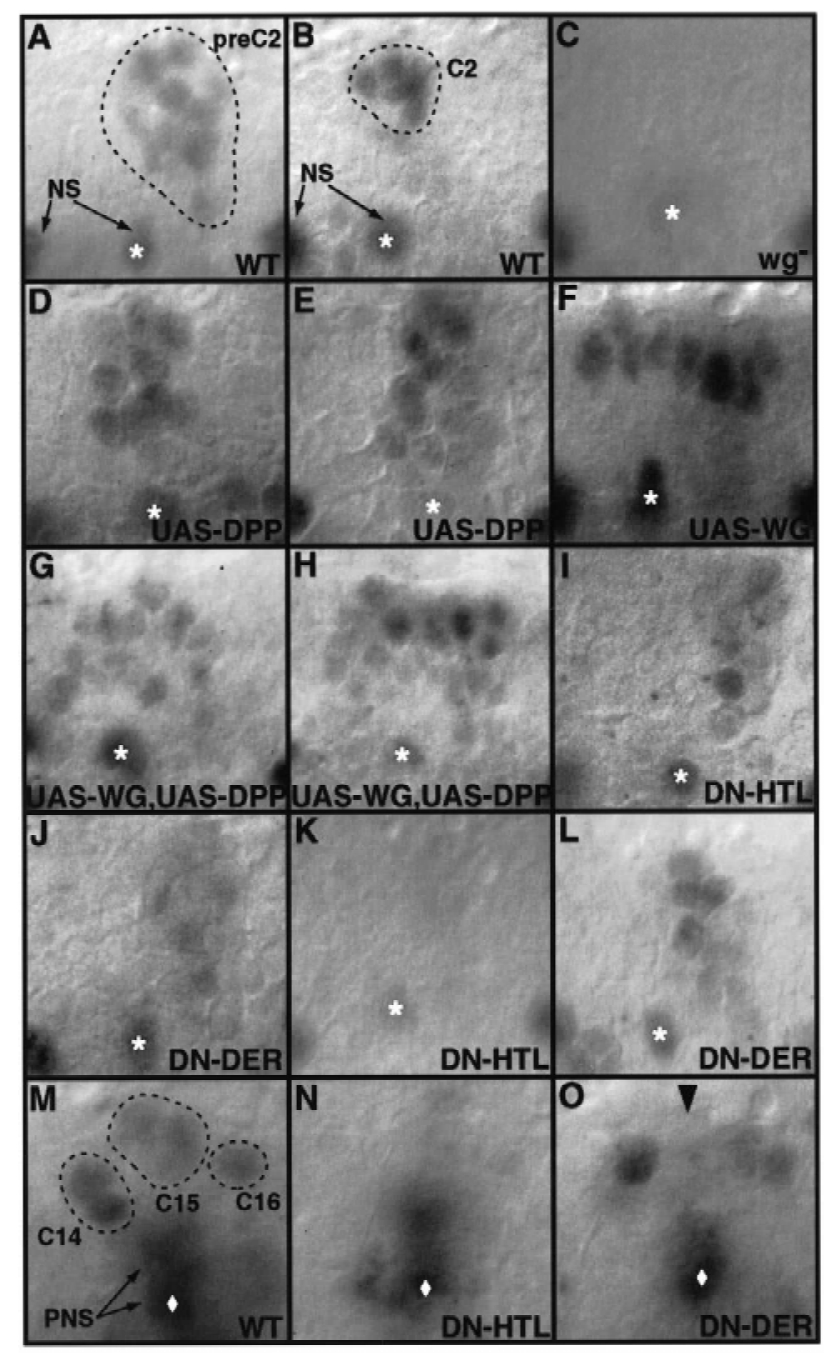

mesodermal organization (data not shown). However, we were able to demonstrate that Dpp is involved in early mesodermal L'sc expression by ectopically expressing Dpp using the Gal4-UAS system (Brand and Perrimon 1993). twi-Gal4-mediated targeting of Dpp had no effect on the formation of preC2 (Fig. 2D) but did prolong the L'sc expression in the ventralmost cells of preC2 (Fig. 2, cf. B and E). The ventral extent of L'sc expression was not expanded, but rather ended at the lateral gap that normally forms in the $\mathrm{Wg}$ stripe during stage 10 (data not shown). This suggests that L'sc is induced in mesodermal cells that are exposed to both Wg and Dpp. Consistent with this hypothesis, ectopic Wg induced additional L'sc expression along the anteroposterior axis of the embryo, but only in dorsal mesodermal cells that are confined to the Dpp domain (Fig. 2F).

As a further test of the dual requirement for $\mathrm{Wg}$ and Dpp for mesodermal L'sc expression, we ectopically expressed both signaling molecules together. This resulted in an anteroposterior extension of L'sc in both preC2 and $\mathrm{C} 2$ due to ectopic $\mathrm{Wg}$, as well as the persistence of $\mathrm{L}$ 'sc in the ventral portion of the precluster at the $\mathrm{C} 2$ stage due to ectopic Dpp (Fig. 2G,H). These findings demonstrate
Figure 2. Dependence of mesodermal L'sc expression on $\mathrm{Wg}$, Dpp, $\mathrm{Htl}$, and DER. $(A) \mathrm{L}$ 'sc expression in preC2 in a single hemisegment of a late stage 10 wild-type embryo. Additional L'sc expression occurs in the nervous system (NS). (B) L'sc expression in a C2. $(C)$ PreC2 is completely missing in a homozygous $w^{C X 4}$ mutant embryo. Ventral preC1, as well as most dorsal and ventral L'sc clusters, are also $w g$ dependent (data not shown). (D) Ectopic expression of Dpp driven by $t w i-G a 14$ does not affect preC2 formation. $(E)$ Ectopic Dpp causes L'sc to be maintained in all cells of preC2 when restriction to $\mathrm{C} 2$ normally occurs. $(F)$ Ectopic Wg induces L'sc in dorsal mesodermal cells both anterior and posterior to a normal C2. Ectopic Wg plus Dpp causes an anteroposterior extension of the L'sc domain at the preC2 stage $(G)$, and persistence of an enlarged preC2 at the C2 stage $(H)$. Neither $t w i-G a l 4$ plus Dmef2-Gal4-induced expression of dominant-negative Htl $(I)$ nor twi-Gal4-mediated expression of dominant-negative DER $(J)$ affects formation of preC2. Dominant-negative $\mathrm{Htl}(K)$ but not dominant-negative DER $(L)$ blocks $\mathrm{C} 2$ formation. $(M)$ L'sc expression in C14-C16 in an earlystage 11 wild-type embryo. (N) Formation of C14, C15, and $\mathrm{C} 16$ is inhibited by dominant-negative $\mathrm{Htl}$. (O) Formation of $\mathrm{C} 15$, but not C14 or C16, is inhibited by dominant-negative DER. (Arrowhead) The position of the missing C15. The asterisks in $A-L$ indicate the position of a nervous system progenitor that serves as a reference point for comparing the various mesodermal L'sc patterns. The diamonds in $M-O$ mark peripheral nervous system (PNS) cells in later stage embryos.

that the mesodermal prepattern represented by early L'sc expression is established by the combined activities of $\mathrm{Wg}$ and Dpp. However, additional factors must be involved, because ectopic Wg and Dpp did not induce panmesodermal L'sc expression at the precluster stage.

\section{Signaling by EGF and FGF receptors induces the formation of mesodermal equivalence groups} within the territory prepatterned by Wg and Dpp

In addition to $\mathrm{Wg}$ and Dpp, signaling by the Drosophila EGF and Htl FGF receptors is required for the determination of a subset of muscle and cardiac progenitors, including those expressing Eve (Buff et al. 1998; Michelson et al. 1998). We were interested, therefore, in whether DER and $\mathrm{Htl}$ are also involved in the formation of L'sc preclusters or clusters. To examine this question, we targeted expression of dominant-negative forms of both receptors to the embryonic mesoderm. Neither dominantnegative Htl (Fig. 2I) nor dominant-negative DER (Fig. 2J) affected preC2 development. However, inhibition of Htl, but not DER, signaling led to loss of C2 (Fig. 2K,L). This requirement for $\mathrm{Htl}$ reflects a direct involvement in cell 
fate specification because mesoderm migration is completely normal when dominant-negative $\mathrm{Htl}$ is expressed under the present conditions (Michelson et al. 1998).

After the singling out of $\mathrm{P} 2, \mathrm{C} 15$ forms at the position occupied previously by $\mathrm{C} 2$ (Figs. $1 \mathrm{~K}$ and $2 \mathrm{M}$ ), a process that requires both DER and $\mathrm{Htl}$ (Fig. 2N,O), in agreement with our prior demonstration that both RTKs are required for muscle DA1 development (Buff et al. 1998; Michelson et al. 1998). Htl (but not DER) also contributes to the formation of other dorsal L'sc clusters (Fig. $2 \mathrm{~N}, \mathrm{O}$ ). In summary, establishment of the L'sc prepattern does not require the activity of either DER or Htl. However, both RTKs are essential for the subsequent organization of L'sc equivalence groups within the mesodermal territory prepatterned by Wg and Dpp.

\section{MAPK is locally activated within mesodermal equivalence groups and persists in singled out progenitors}

Transduction of RTK signals occurs, at least in part, via the Ras/MAPK cascade (van der Geer et al. 1994; Seger and Krebs 1995). By use of an antibody that is specific for the diphosphorylated or activated form of mitogen-associated protein kinase (MAPK) (diphospho-MAPK), it is possible to identify localized sites of RTK signaling in the Drosophila embryo (Gabay et al. 1997a,b). We have used this reagent to monitor the spatial and temporal involvement of DER and Htl in the formation of mesodermal L'sc clusters and the specification of the corresponding progenitors.

The earliest activation of MAPK in the fully migrated mesoderm occurs in $\mathrm{C} 2$, coincident with the restriction of L'sc and prior to the appearance of Eve in this cluster (Fig. 3A; data not shown). These findings are consistent with the requirement demonstrated previously of $\mathrm{Htl}$ for C2 development. Diphospho-MAPK persists in C2 after the onset of Eve expression (Fig. 3B), but fades from most C2 cells during progenitor selection (Fig. 3C). Activated MAPK remains in P2 transiently and then disappears (Fig. 3D,E). Simultaneously, C15 begins to express both diphospho-MAPK and Eve (Fig. 3F). The activation of MAPK in C15 is expected from the involvement of $\mathrm{Htl}$ and DER in C15 formation (Fig. 2N,O). P2 then divides to yield sibling founder cells, neither of which initially contains activated MAPK (Fig. 3G; F2s). However, by the time P15 is singled out, MAPK is reactivated in one of the sibling F2s (Fig. 3H). As is the case with P2, diphospho-MAPK remains at high levels in P15 (Fig. 3F,H). Moreover, the persistence of MAPK activation correlates with the maintenance of Eve expression in both progenitors (Fig. 3D,H). Additional diphospho-MAPK expression is observed in cells derived from C14, C16, and C17 (Fig. $3 \mathrm{G}, \mathrm{H})$, in agreement with the involvement of $\mathrm{Htl}$ in the formation of these clusters (Fig. 2N). Thus, both the temporal and spatial patterns of diphospho-MAPK expression are consistent with a requirement for RTK signaling in the formation of mesodermal L'sc clusters, the induction of mesodermal Eve expression, and the singling out of muscle and cardiac progenitors.

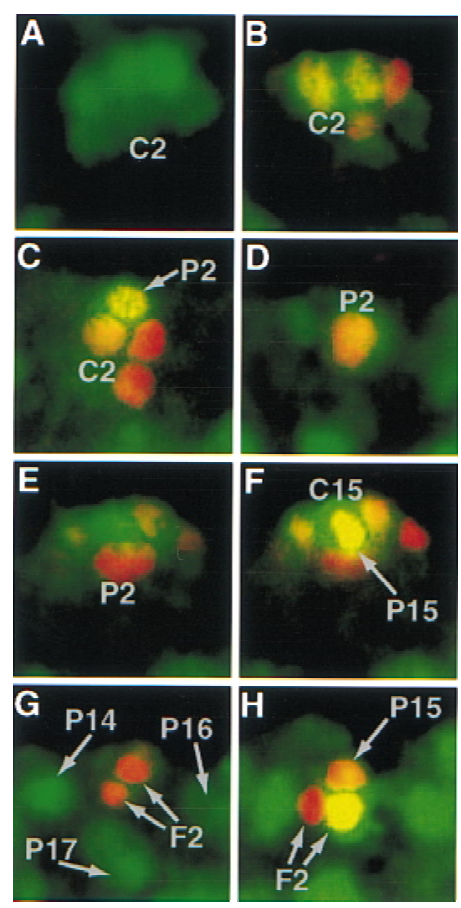

Figure 3. In situ localization of activated MAPK during the specification of mesodermal Eve progenitors. Wild-type embryos were stained for expression of diphospho-MAPK (green) and Eve (red). (A) At late-stage 10, diphospho-MAPK first appears prior to Eve in all C2 cells. (B) Eve initiates in C2 and diphospho-MAPK begins fading from some $\mathrm{C} 2$ cells. $(C)$ Diphospho-MAPK continues to decline in most $\mathrm{C} 2$ cells with the exception of progenitor $2(\mathrm{P} 2)$. Eve has not yet restricted to P2. $(D)$ P2 has been singled out and retains both Eve and diphosphoMAPK. (E) Eve persists in P2 although diphospho-MAPK is no longer detected. $(F)$ Eve and diphospho-MAPK are coexpressed in $\mathrm{C} 15$ with the highest levels of both proteins in P15. (G) Sibling F2 founders express Eve but not diphospho-MAPK after P2 divides. Additional diphospho-MAPK occurs in P14, P16, and P17. $(H)$ Diphospho-MAPK is reactivated in one F2 founder as P15 is singled out. The indicated positions of P15 and F2 are consistent with L'sc plus Eve double labeling (see Fig. 1L,M). As with F2, MAPK is reactivated in only one F15 (data not shown).

\section{Ras1 signaling specifies mesodermal progenitors and promotes founder cell differentiation}

We have shown previously that constitutive activity of DER, Htl, or Ras1 is associated with the development of supernumerary Eve-expressing mesodermal cells (Fig. 4A,C; Gisselbrecht et al. 1996; Buff et al. 1998; A.M. Michelson and S. Gisselbrecht, unpubl.). However, these earlier experiments did not establish whether this response is due to activated Ras1-induced proliferation of normal Eve cells or due to the recruitment of additional cells to an Eve-positive fate. Activated Ras1 does not increase the sizes of L'sc clusters (data not shown), suggesting that Ras1 is not simply stimulating the division of mesodermal cells. However, to definitively address this question, we examined the effects of constitutive Ras1 activity in string (stg) mutant embryos. Because 


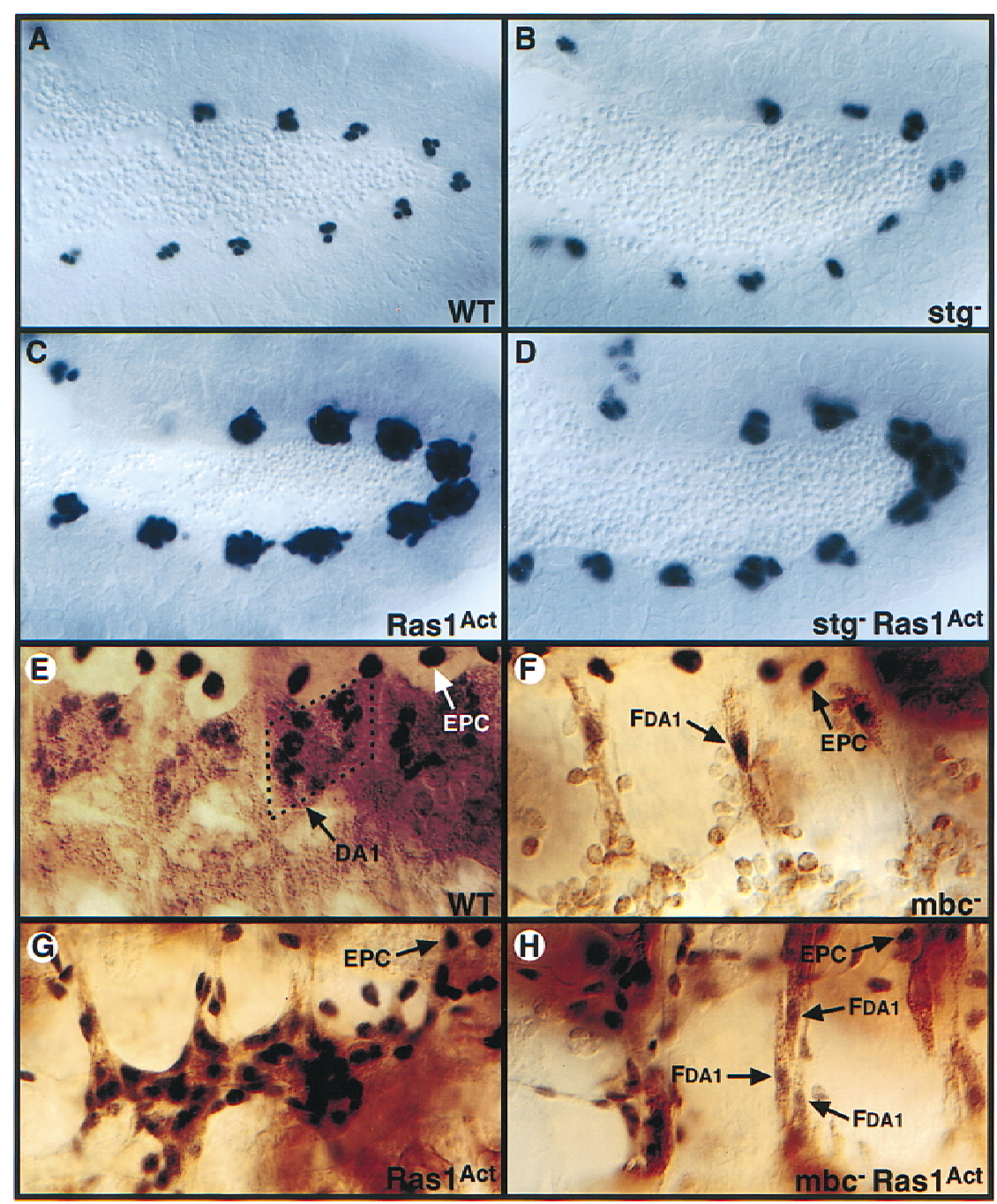

Figure 4. Effect of constitutive Ras1 signaling on the specification of mesodermal progenitors and the differentiation of muscle founders. $(A-D)$ Stage 11 embryos of the indicated genotypes were stained for Eve expression. $(E-H)$ Stage 16 embryos were stained for Eve (black) and myosin heavy chain (brown) expression. (A) Three to four Eve-positive cells are observed in each wildtype hemisegment. These represent sibling F2 founders plus either a single P15 progenitor or the sibling cells derived from P15 division. (B) In a stg mutant, one to two Eve progenitors form per hemisegment. Constitutive Ras1 activation generates additional Eve-positive mesodermal cells in otherwise wild-type $(C)$ or stg mutant $(D)$ embryos. $(E)$ Eve is expressed in two pericardial cells (EPC) and one dorsal somatic muscle (DA1) in each wild-type hemisegment. $(F)$ Muscle fusion does not occur in mbc mutant embryos and muscle founders expressing Eve $\left(\mathrm{F}_{\mathrm{DA} 1}\right)$ can be identified by their spindleshaped morphology. (G) Constitutive Ras1 activation induces the formation of large syncytia containing multiple Eve-positive nuclei. $(H)$ Extra Eve-expressing muscle founders are present in $m b c$ mutant embryos when Ras1 is constitutively activated. strong alleles of stg prevent all post-blastoderm cell divisions (Edgar and O'Farrell 1989), a potential cell-proliferation effect of activated Ras1 should be blocked in this genetic background. On the other hand, stg should not inhibit the overproduction of Eve-expressing cells if Ras 1 promotes their determination. In a stg mutant, only one to two Eve-positive cells are present in each hemisegment (Fig. 4B). These cells correspond to the Eve progenitors that form in wild-type embryos (P2 and P15 in Figs. 1J,L and 3E,H). The presence of only one Eve progenitor in some segments of stg mutant embryos presumably is due to the smaller number of mesodermal cells that contribute to L'sc clusters when zygotic cell divisions do not occur. Significantly, activated Ras1 generates more Eve progenitors in a stg mutant than are seen in the mutant alone (Fig. 4B,D). We conclude that Ras1 promotes the formation of additional Eve progenitors by inducing more mesodermal cells to assume this fate and not by stimulating the normal progenitors to divide.

Next, we assessed whether the Ras1 pathway is sufficient to induce progenitor differentiation by examining the myogenic effects of Ras1 at later developmental stages in both wild-type and myoblast city $(\mathrm{mbc})$ mutant embryos. In the absence of $m b c$ function, muscle fusion does not occur and differentiated muscle founders appear as spindle-shaped myoblasts that express myosin and founder cell markers such as Eve (Fig. 4E,F; Rushton et al. 1995). In contrast to the mbc mutant in which one Eve-expressing muscle DA1 founder is present in each hemisegment, multiple such cells form in $m b c$ embryos under the influence of activated Ras1 (Fig. 4H). All of the Eve plus myosin-positive myoblasts seen in these embryos have the elongated morphology of muscle founders, as opposed to the round shape of neighboring Eve-negative myoblasts. Large syncytia containing many Eve-positive nuclei are present when Ras1 is activated in a wild-type background (Fig. 4G). Although extra Eve pericardial cells initially appear under the influence of activated Ras1 (data not shown), Eve expression in such cells is lost by later stages (Fig. 4G,H). We conclude that ectopic activation of the Ras1 pathway not only stimulates Eve expression in additional progenitors, but also promotes the formation and differentiation of supernumerary muscle founders. 
The entire mesodermal domain prepatterned by $W g$ and Dpp is competent to respond to Ras1 activation

The extra Eve-positive cells induced by activated Ras1 are confined to clusters that have anteroposterior and dorsoventral boundaries resembling those of L'sc precluster 2. The anteroposterior dimensions of these enlarged Eve clusters align precisely with the borders of the Wg stripe (Fig. 5A,B). Furthermore, activated Ras1 prematurely induces Eve expression in all preC2 cells (Fig. 5C). This Eve expression occurs earlier than usual because, in these experiments, constitutive Ras1 expression is driven by twi-Gal4, which initiates at stage 7 (Greig and Akam 1993), prior to the normal activation of MAPK in the dorsal mesoderm at late stage 10 (Fig. 3A). By the time L'sc is restricted to a single P2 in wild-type embryos, at least two to three such cells are observed in activated Ras1 embryos (data not shown). When C14C17 normally appear, activated Ras1 induces the coexpression of L'sc and Eve in a large group of cells that encompasses all of C15, as well as most of C14, C16, and C17 (Fig. 5D). Many of these cells retain L'sc longer than normal and become progenitors that divide to produce extra Eve-expressing founders (Fig. 5E,F). Thus, delocalized activation of the Ras/MAPK pathway induces progenitor-specific gene expression in the entire mesodermal domain prepatterned by Wg and Dpp.

The singling out of progenitors from mesodermal equivalence groups depends on lateral inhibition mediated by the neurogenic genes (Corbin et al. 1991; Bate et al. 1993; Carmena et al. 1995; Baker and Schubiger 1996). In the absence of $D 1$ function, L'sc and Eve initiate at the correct time and in the normal number of cells in both C2 and C15 (Fig. 5G,H). Unlike in wild type, all C2 cells in the Dl mutant initially develop like progenitors by acquiring high levels of L'sc and Eve. However, this expression is transient in the neurogenic mutant; both markers disappear from most C2 cells prior to the formation of C15 (data not shown), consistent with the loss of pericardial cells from neurogenic mutants documented previously (Hartenstein et al. 1992). In contrast, all C15 cells persist as Eve progenitors in a Dl mutant embryo (Fig. 5I,J). The effects of constitutive Ras1 and of D1 loss-of-function are shown schematically in comparison to the normal progression of progenitor specification in Figure $5 \mathrm{~K}$. Whereas the entire prepatterned region is competent to respond to RTK/Ras signaling, Dl only mediates cell fate decisions within an individual equivalence group. In addition, neither Ras1 nor neurogenic signaling affects L'sc precluster formation.

Wg and Dpp are required for the mesodermal function of Ras 1

All preC2 cells develop as Eve progenitors in response to activated Ras1. Because preC2 is dependent on $\mathrm{Wg}$ and Dpp for its formation, we investigated whether Wg and Dpp are required for the mesodermal effects of Ras1. Such epistasis experiments are possible because gain of Ras 1 function causes an overproduction of Eve progeni-

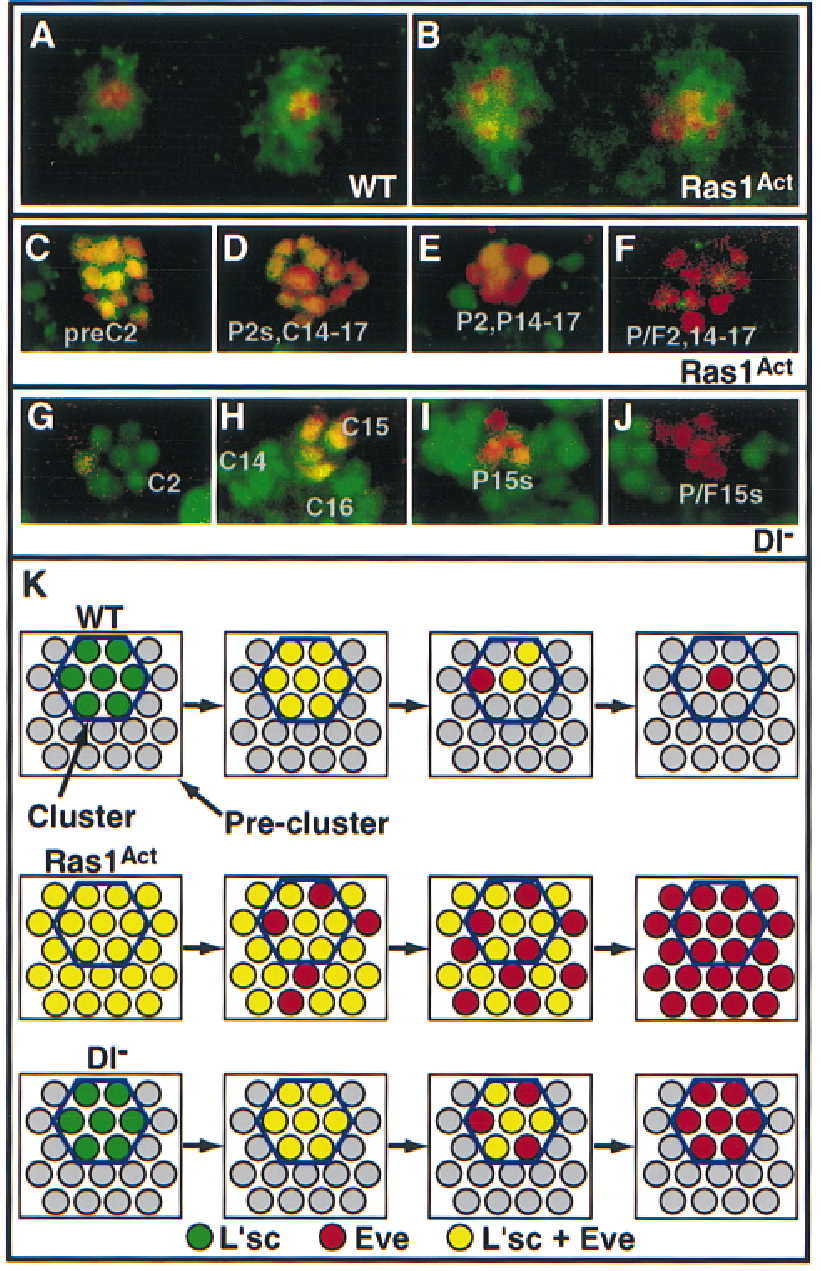

Figure 5. Comparison between the mesodermal effects of Ras1 gain-of-function and neurogenic gene loss-of-function. $(A, B)$ Stage 11 embryos were stained for expression of $\mathrm{Wg}$ (green) and Eve (red). (C-J) Stage 10-11 embryos were stained for expression of L'sc (green) and Eve (red). (A) Mesodermal Eve cells develop in the $\mathrm{Wg}$ domain in wild-type embryos. $(B)$ The additional mesodermal Eve cells induced by constitutive Ras 1 are confined to the Wg stripe. $(C)$ Activated Ras1 induces the premature expression of Eve in all preC2 cells. (D) After several P2s are specified, activated Ras 1 causes Eve and L'sc to be coexpressed in C15 and in most (or, as in this case, all) cells of C14, C16, and C17. $(E, F)$ Additional Eve progenitors and founders derived from $\mathrm{C} 2$ and C14-C17 are produced under the influence of activated Ras1. Separate L'sc clusters cannot be resolved in $D-F$ because L'sc expression is prolonged in each by activated Ras1. Initial C2 formation is normal in a $D 1$ mutant embryo $(G)$, and Eve is activated in $\mathrm{C} 15$, in which it is initially coexpressed with L'sc as in wild type $(H)$. $(I)$ By the time L'sc and Eve normally become restricted to a single progenitor, all $\mathrm{C} 15$ cells retain expression of both markers in a Dl mutant. (J) Extra C15-derived Eve cells persist in a $D l$ mutant. $(K)$ Schematic representation of L'sc and Eve expression in wild type, Dl mutant, and embryos expressing an activated form of Ras1. See text for details.

tors (Figs. 4C and 5B-F), whereas these cells are completely missing from $w g$ and $d p p$ mutant embryos (Fig. 6A,B; Frasch 1995; Wu et al. 1995). In either a $w g$ or $d p p$ 


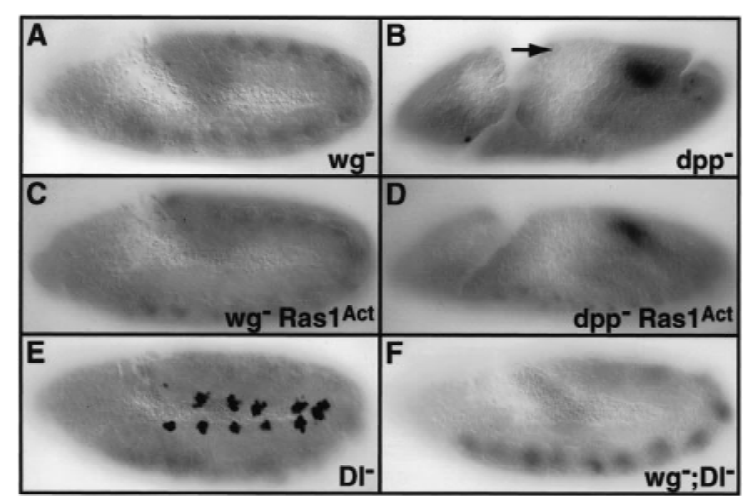

Figure 6. Epistatic relationships among the positive and negative signaling pathways involved in the specification of mesodermal Eve progenitors. Stage 11 embryos of the indicated genotypes were stained for expression of Eve. $(A)$ In a wg mutant, no mesodermal Eve progenitors develop. $(B)$ Loss of $d p p$ function causes strong ventralization of the embryo and abnormal germband extension. However, mesodermal cells migrate in these embryos such that the dorsal margin of the mesoderm occupies the position indicated by the arrow. No Eve expression is detected at this or any other location in the mesoderm of $d p p$ mutant embryos. $(C)$ Constitutive Ras1 activation does not induce mesodermal Eve progenitors in the absence of $w g$ function. (D) Loss of $d p p$ function blocks the ability of activated Ras1 to generate mesodermal Eve progenitors. (E) Additional Eve progenitors form in a $D 1$ mutant embryo. $(F)$ No mesodermal Eve progenitors develop in a $w g ; D 1$ double mutant.

mutant background, the effect of activated Ras1 on mesodermal Eve expression is completely blocked (Fig. $6 \mathrm{C}, \mathrm{D})$. That is, the specification of Eve progenitors requires Wg and Dpp even when the Ras1 pathway is functioning constitutively.

\section{$W g$ is epistatic to the mesodermal function of Dl}

Given the opposite mesodermal Eve phenotypes associated with loss of $w g$ and $D l$ functions, we examined the epistatic relationship between these positive and negative signals. Although loss of $D l$ function results in an overproduction of Eve progenitors (Figs. 5I,J and 6E) and loss of $w g$ function prevents mesodermal Eve expression (Fig. 6A), a $w g ; D l$ double-mutant embryo has an Eve expression pattern identical to that of a wg mutant (Fig. $6 \mathrm{~F})$. Thus, formation of Eve progenitors requires the positive influence of $\mathrm{Wg}$ even in the absence of the inhibitory signal mediated by the neurogenic genes.

\section{Wg, Dpp, and Ras1 are sufficient for the specification of mesodermal Eve progenitors}

Our data suggest that the Wg, Dpp, and RTK/Ras pathways cooperate to induce mesodermal Eve progenitors. We tested this hypothesis by comparing the effects on progenitor specification of ectopically expressing these three signals either alone or in all possible combinations. Misexpression of Wg and/or Dpp under twi-Gal4 control does not stably alter the number of dorsal P2 or P15 Eve progenitors (Frasch 1995; Lawrence et al. 1995; data not shown). However, ectopic Dpp, either by itself or together with $\mathrm{Wg}$, induces Eve in a ventral mesodermal progenitor (corresponding to a normal P1 or P6) that normally lacks Eve expression (Fig. 7A-C). The latter cell expresses activated MAPK, it falls within the Wg domain, and its transformation under the influence of Dpp is also $\mathrm{Wg}$ dependent (data not shown). Thus, $\mathrm{Wg}$, and Dpp are both necessary, but not sufficient, for the formation of dorsal Eve progenitors, and the induction of a ventral Eve progenitor is coincident with $\mathrm{Wg}$, Dpp, and MAPK signals.

In contrast, ectopic Wg plus activated Ras1 induces additional Eve progenitors in the normal Wg interstripe region. L'sc also is expressed ectopically in this area (Fig. $7 \mathrm{D}, \mathrm{E})$. However, all of the extra Eve progenitors induced by ectopic Wg plus activated Ras1 are dorsally restricted. A similar synergy was observed between Dpp and activated Ras1. In this case, Eve and L'sc expression extends along the dorsoventral axis (Fig. 7F,G). A striking feature of this response is the restriction of the additional Eve/ L'sc-expressing cells to stripes that fall within the $\mathrm{Wg}$ domain. Moreover, this effect of ectopic Dpp plus activated Ras1 absolutely requires Wg activity (data not shown).

Finally, we found that Wg, Dpp, plus activated Ras1 are sufficient for the induction of mesodermal Eve expression. Simultaneous ectopic expression of all three signals results in the induction of Eve and L'sc in cells of the lateral and ventral mesoderm that lie outside of the normal Wg and Dpp domains (Fig. 7H,I). Not every mesodermal cell assumes an Eve-positive fate under these conditions; the majority that fail to do so fall in the normal low Twi domain (Dunin Borkowski et al. 1995; see Discussion). In summary, our results indicate that $\mathrm{Wg}$, Dpp, and RTK/Ras1 signals are both necessary and sufficient for establishing an Eve-positive mesodermal progenitor cell fate.

\section{Discussion}

We have demonstrated that an early event in the differentiation of the Drosophila embryonic mesoderm is the generation of a prepattern represented by expression of the proneural gene, l'sc. In the dorsal region of the embyro, this L'sc prepattern is both delimited by and dependent on the intersecting domains of Wg and Dpp expression. Signaling by two RTKs is superimposed on this mesodermal prepattern to generate equivalence groups from which specific muscle and cardiac progenitors are singled out. The final progenitor pattern is dictated by the concerted deployment of these multiple intercellular signals.

\section{A model for combinatorial signaling by $W g, D p p$,} and RTK/Ras pathways in mesoderm development

A model for combinatorial signaling in embryonic mesoderm development is presented in Figure 8. According 
Figure 7. Sufficiency of $\mathrm{Wg}, \mathrm{Dpp}$, and Ras1 signals for the specification of a mesodermal Eve cell fate. Stage 11 embryos were stained for expression of Eve alone $(A, B, D, F, H)$ or Eve (red) plus L'sc (green) $(C, E, G, I)$. (A) Ventral view of a wild-type embryo showing Eve expression in the central nervous system (CNS). No Eve is present in the ventral or lateral mesoderm. $(B, C)$ Ectopic Dpp induces Eve expression in ventral mesodermal cells that correspond to wild-type P1 or P6. The embryo in $B$ is slightly older and so has more than one Eve-positive ventral mesodermal cell per hemisegment due to progenitor division. The arrowhead in $C$ points to Eve expression in the CNS. Formation of dorsal mesodermal Eve clusters and progenitors is unaffected by ectopic Dpp. $(D, E)$ Ectopic Wg plus activated Ras 1 induces the formation of additional Eve progenitors along the anteroposterior axis in the regions between the normal Eve clusters (arrowheads). The approximate locations of the extra progenitors induced by activated Ras 1 alone are indicated by the dotted circles. (as) Amnioserosa. $(F, G)$ Ectopic Dpp plus activated Ras1 generates extra Eve progenitors in a stripe that extends from $\mathrm{C} 15$ dorsally to $\mathrm{C} 1 /$ C6 ventrally (brackets). (H,I) Ectopic Wg plus Dpp plus activated Ras1 causes Eve progenitors to form in regions that normally are never exposed to either Wg or Dpp (arrowheads). The embryos in $D, F$, and $H$ contain Eve founders as well as progenitors, as they are slightly older than those in $E, G$, and $I$.

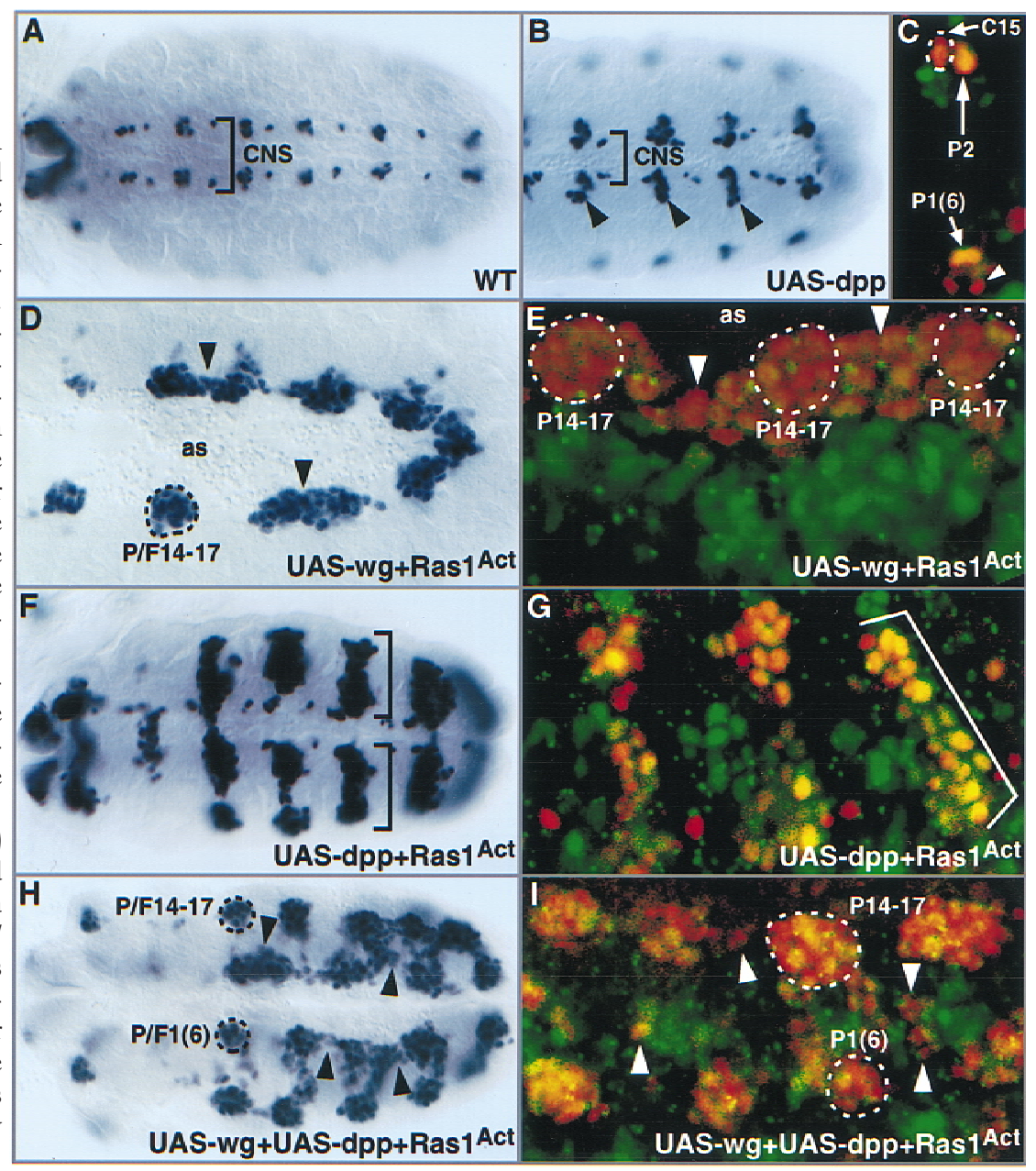

to this scheme, the combined influence of Wg and Dpp generates a broad region of L'sc expression that we refer to as a precluster. This region encompasses L'sc clusters 2 and 15 in their entirety, and includes those cells of C14, C16, and C17 that are closest to C2 and C15 (Carmena et al. 1995). All cells within this prepatterned area are competent to respond to a subsequent Ras1 signal. However, the Ras1 pathway only functions in subsets of precluster cells due to the localized activation of $\mathrm{Htl}$ (for C2) or DER plus Htl (for C15). RTK signaling stabilizes
L'sc expression in discrete clusters of cells and later induces Eve expression throughout a cluster. This initial uniform response of an entire cluster is the hallmark of a group of developmentally equivalent cells (Greenwald and Rubin 1992). Finally, the inhibitory influence of the neurogenic genes opposes the combined activities of the three positive signals to facilitate the specification of a single Eve progenitor.

Our results are in complete agreement with the various features and predictions of this model, and can be

Figure 8. A model for the combinatorial effects of Wg, Dpp, Ras1, and neurogenic signals in embryonic mesoderm development. The intersection of $\mathrm{Wg}$ (red) and Dpp (blue) expression domains delineates a prepattern (purple) in which L'sc is initially expressed as a precluster (green). This entire precluster is competent to respond to a subsequent RTK/Ras1 signal. However, localized activation of $\mathrm{Htl}$ and DER restricts L'sc to a subset of precluster cells that represent an

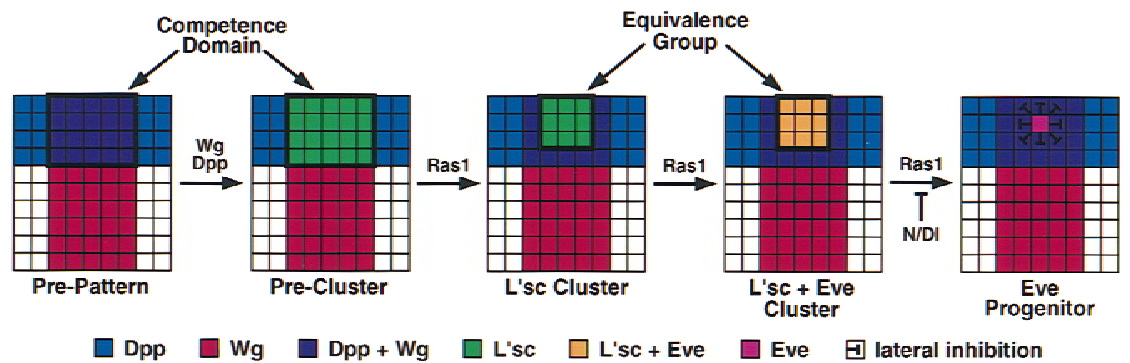
equivalence group. Further RTK/Ras1 signaling activates Eve expression in all cells of the L'sc cluster (orange). A single progenitor is determined under the lateral inhibitory influence of the neurogenic genes (pink). This model applies to both the pericardial and somatic muscle Eve/L'sc clusters, both of which derive from the same precluster. For simplicity, only one cluster is shown. 
summarized as follows. (1) The prepattern represented by L'sc precluster 2 has anteroposterior and dorsoventral boundaries that correlate precisely with the intersection of the Wg and Dpp domains. (2) PreC2 formation is dependent on $\mathrm{Wg}$ and its borders are predictably altered by ectopic expression of Wg and Dpp. (3) Inhibition of $\mathrm{Htl}$ or DER function does not interfere with the L'sc prepattern. However, $\mathrm{Htl}$ is required for $\mathrm{C} 2$ formation, and both $\mathrm{Htl}$ and DER are necessary for C15. (4) RTK/Ras1 signaling is essential for Eve expression in $\mathrm{C} 2, \mathrm{C} 15$, and their progenitors. Furthermore, constitutive activation of Ras 1 is sufficient to induce Eve in all cells of preC2, indicating that all preC2 cells are competent to respond to RTK/ Ras1 signaling. (5) The spatial expression of activated MAPK confirms that RTK signaling is localized during equivalence group formation. (6) Consistent with $\mathrm{Wg}$ and Dpp conferring on mesodermal cells the competence to respond to an instructive Ras1 signal, activated Ras1 fails to induce Eve expression in $w g$ and $d p p$ mutant embryos. Moreover, the combined activities of all three signals are sufficient for the induction of a mesodermal Eve cell fate. (7) Loss of neurogenic gene function causes all cells of a L'sc cluster to become progenitors, as expected for cells in an equivalence group (Greenwald and Rubin 1992). In addition, the positive Wg signal is still required for progenitor specification in the absence of Dl function. (8) The proposed temporal order of signaling events is consistent with the timing of $\mathrm{Wg}$, Dpp, and diphospho-MAPK expression and with the phenotypic effects associated with altering each of these signals.

A salient feature of our model is that equivalence groups form in response to the localized functions of two RTKs acting within the larger prepatterned region. The mesodermal diphospho-MAPK expression pattern not only provides strong support for this hypothesis, but also raises the question of how the upstream receptors are activated in discrete subsets of competent cells. For C15, this may occur through induction by $\mathrm{P} 2$, which expresses Rhomboid, a factor that can nonautonomously stimulate DER in adjacent cells (Golembo et al. 1996; Buff et al. 1998). The localized activation of Htl could result from restricted expression of its as yet unidentified ligand. Htl itself is enriched in groups of Eve-positive mesodermal cells, a process that might also contribute to the Htl-dependent formation of these clusters (Michelson et al. 1998).

Wg and Dpp are instructive for precluster formation but are only permissive for the induction of Eve progenitors. However, this permissive function is absolutely required because the mesodermal effect of activated Ras1 is blocked in $w g$ and $d p p$ mutant embryos. Thus, the $\mathrm{Wg}$, Dpp, and Ras1 pathways are not acting in a strictly sequential manner to specify Eve progenitors.

Both P2 and P15 express Eve, form in close proximity to each other, and are specified by related signaling mechanisms, yet they differentiate into distinct structures. The disparate developmental fates of these progenitors may be determined by the different RTKs involved in their formation. Whereas $\mathrm{P} 2$ requires only $\mathrm{Htl}$, both Htl and DER are essential for P15 (Buff et al. 1998;
Michelson et al. 1998; this paper). Distinct downstream signaling pathways, or different quantitative outputs of the same signal transducers, could explain the unique responses mediated by these two RTKs (Marshall 1995; Clandinin et al. 1998). The existence of a requisite RTK signal threshold for Eve expression is suggested by the behavior of L'sc clusters 14 and 16. Although C14 and C16 are Htl dependent, and at least some cells of both clusters lie within the Wg/Dpp domain, neither cluster expresses Eve unless Ras1 is ectopically activated. Different DER activity thresholds may also exist for the formation of other embryonic muscles (Buff et al. 1998).

The concept of developmental prepatterns has been considered for many decades (Stern 1954). The existence of a prepattern is typically inferred from the identification of a broad region that is competent to assume the fate that only one or a few cells normally adopt. We have shown that the intersecting expression of Wg and Dpp create a mesodermal prepattern. Because the mechanisms responsible for the $\mathrm{Wg}$ and Dpp expression patterns have been traced to the maternal systems that initiate formation of the basic body plan of the Drosophila embryo (Lawrence 1992), our findings provide a direct link between the earliest developmental processes and the later differentiation of specific tissues.

\section{Opposing activities of positive and negative signals involved in progenitor specification}

L'sc and Eve are initially activated in all cells of C2 and C15 through the concerted functions of Wg, Dpp, and Ras1. However, the inhibitory influence of the neurogenic genes insures that only a single progenitor retains expression of these markers. Neurogenic signaling thus opposes the positive influence of the $\mathrm{Wg}$, Dpp, plus RTK/Ras pathways. One scenario for these opposing functions involves the sequential, nonoverlapping activities of the positive and negative signals. However, arguing against such a mechanism is our observation that diphospho-MAPK persists after cluster formation and becomes progressively restricted to a single progenitor. This implies that RTK signaling is coincident with Notch-mediated lateral inhibition and suggests that progenitor specification involves a competitive or antagonistic interaction between the Ras and Notch pathways.

\section{Interplay between signaling molecules and nuclear determinants of mesodermal fate}

Several transcription factors with important functions in mesoderm development have been identified, including Twi and Tin. In addition to its early role in mesoderm determination, later fluctuations in Twi expression levels subdivide the mesoderm (Dunin Borkowski et al. 1995; Baylies and Bate 1996). Eve progenitors are normally found in the high Twi domain (Dunin Borkowski et al. 1995), which may explain why the most robust activation of Eve by ectopic Wg, Dpp, and Ras1 is confined to cells having high Twi levels. 
Tin also subdivides the mesoderm by determining dorsal mesodermal identities, including those characterized by Eve expression (Azpiazu and Frasch 1993; Bodmer 1993). In addition to a requirement for appropriate levels of Twi, Tin must be present for Ras1 to induce the formation of Eve progenitors (Michelson et al. 1998). As dorsal Tin expression is Dpp-dependent (StaehlingHampton et al. 1994; Frasch 1995), Tin can be considered a downstream component of the Dpp pathway that is involved in Eve progenitor specification. Thus, transcription factors intrinsic to the mesoderm, but controlled in part by the extrinsic signals analyzed here, make critical contributions to the combinatorial model for progenitor specification.

\section{Multiple roles of mesodermal RTK signaling}

RTK signaling in the Drosophila embryonic mesoderm is important for cell migration (Beiman et al. 1996; Gisselbrecht et al. 1996; Shishido et al. 1997) and cell fate specification (Lüer et al. 1997; Buff et al. 1998; Michelson et al. 1998). We have shown here that the latter role can be divided into several distinct functions. First, Htl and DER promote the formation of L'sc clusters or equivalence groups. Second, Ras1 signaling activates identity gene expression in the entire group of equivalent cells. Third, activated MAPK becomes restricted to progenitors, suggesting a role for RTK signaling in progenitor selection. Fourth, MAPK is reactivated in one of the sibling founders derived from a single progenitor, suggesting that RTK activity helps to establish or maintain the founder identity that is initiated by the asymmetric division of its progenitor (Ruiz Gomez and Bate 1997; Carmena et al. 1998). Alternatively, RTK function in some founder cells could promote their differentiation. The ability of activated Ras1 to generate supernumerary Eve founders in $m b c$ mutant embryos is consistent with this last possibility. Interestingly, the RTK/Ras1 pathway is similarly utilized in a sequential manner during development of the Drosophila compound eye (Freeman 1996).

\section{Concluding remarks}

When analyzed separately, the Wg, Dpp, DER, and Htl pathways are each responsible for the formation of large subsets of mesodermal cells (Staehling-Hampton et al. 1994; Baylies et al. 1995; Frasch 1995; Wu et al. 1995; Ranganayakulu et al. 1996; Buff et al. 1998; Michelson et al. 1998). However, the generation of such cell-type diversity poses a considerable challenge for any one signaling system. One solution to this problem is for each mesodermal progenitor to be specified by a unique combinatorial signaling code, as we have demonstrated for two cellular identities. Although only a limited number of separate signals are available, additional complexity can be generated if each pathway produces a range of specific responses by a graded output mechanism (Katz et al.
1995; Marshall 1995; Buff et al. 1998). Additional flexibility is introduced when the same pathways can either cooperate with or antagonize each other depending on their specific contexts (Jiang and Struhl 1996; this paper). The coordinate effects of Wg, Dpp, and RTK pathways on the development of mesodermal equivalence groups and their progenitors in Drosophila provide one paradigm for how a combinatorial signaling code can manifest its effects. Direct parallels can be drawn to vertebrate myogenesis, in which combinations of related diffusible factors subdivide the developing somite and contribute to the determination of muscle cell fates (Currie and Ingham 1998). An important goal of future studies will be to determine the molecular basis for signal integration in such complex developmental events.

\section{Materials and methods}

Drosophila strains and genetics

The following mutant stocks were used: $\mathrm{wg}^{C X 4}, \mathrm{wg}^{\mathrm{IG} 22}, \mathrm{stg}^{7 B 69}$, $d p p^{H 46}, m b c^{2}, m b c^{3}, D l^{X}$, and $D l^{F X 3}$. Ectopic expression was achieved with the Gal4-UAS system (Brand and Perrimon 1993) and the following fly lines: twi-Gal4 (Greig and Akam 1993; Baylies et al. 1995), Dmef-Gal4 (Ranganayakulu et al. 1996), UAS-Wg (Binari et al. 1997), UAS-Dpp (Staehling-Hampton et al. 1994), UAS-Ras1 ${ }^{\text {Act }}$ (Gisselbrecht et al. 1996; kindly provided by N. Perrimon, Harvard Medical School, Boston, MA), UAS-DNDER (Buff et al. 1998) and UAS-DNHtl (Michelson et al. 1998). Oregon-R was used as the reference wild-type strain. Combinations of mutations and P-element transgenes were generated by standard genetic crosses.

\section{Immunohistochemistry and in situ hybridization}

Embryo fixation, antibody staining, and in situ hybridization were carried out by modifications of standard protocols (Tautz and Pfeifle 1989; Carmena et al. 1995; Gisselbrecht et al. 1996). Antibody against diphospho-MAPK (Gabay et al. 1997a,b) was obtained from Sigma. Both fluorescent and immunohistochemical signals obtained with this antibody were enhanced by use of Tyramide Signal Amplification reagents (New England Nuclear). Microscopy and digital image processing were performed as described previously (Carmena et al. 1995; Gisselbrecht et al. 1996).

\section{Acknowledgments}

We thank the following collegues for providing fly strains, antibodies, and in situ hybridization probes: M. Frasch, R. Nusse, D. Kiehart, W. Gelbart, M. Baylies, N. Perrimon, M. Hoffmann, A. Manoukian, H. Krause, S. Greig, M. Akam, E. Olson, and the Bloomington Drosophila Stock Center. J. Skeath provided invaluable advice on the enhancement of anti-diphospho-MAPK staining. We are grateful to M. Halfon, L. Garcia-Alonso, M. Baylies, and I. Guerrero for helpful discussions and comments on the manuscript. A.C. and F.J. were supported by a predoctoral fellowship and by grants from the Spanish Dirección General de Investigación Científica y Técnica, respectively. A.M.M. is an assistant investigator of the Howard Hughes Medical Institute.

The publication costs of this article were defrayed in part by payment of page charges. This article must therefore be hereby 
marked 'advertisement' in accordance with 18 USC section 1734 solely to indicate this fact.

\section{References}

Azpiazu, N. and M. Frasch. 1993. tinman and bagpipe-two homeobox genes that determine cell fates in the dorsal mesoderm of Drosophila. Genes \& Dev. 7: 1325-1340.

Azpiazu, N., P.A. Lawrence, J.P. Vincent, and M. Frasch. 1996. Segmentation and specification of the Drosophila mesoderm. Genes \& Dev. 10: 3183-3194.

Baker, R. and G. Schubiger. 1995. Ectoderm induces musclespecific gene expression in Drosophila embryos. Development 121: 1387-1398.

-1996. Autonomous and nonautonomous Notch functions for embryonic muscle and epidermis development in Drosophila. Development 122: 617-626.

Bate, M. 1990. The embryonic development of larval muscles in Drosophila. Development 110: 791-804.

- 1993. The mesoderm and its derivatives. In The deve1opment of Drosophila melanogaster (ed. M. Bate and A. Martinez Arias), pp. 1013-1090. Cold Spring Harbor Laboratory, Cold Spring Harbor, NY.

Bate, M. and E. Rushton. 1993. Myogenesis and muscle patterning in Drosophila. C.R. Acad. Sci. Paris 316: 1047-1054.

Bate, M., E. Rushton, and M. Frasch. 1993. A dual requirement for neurogenic genes in Drosophila myogenesis. Dev. Suppl. $149-161$.

Baylies, M.K. and M. Bate. 1996. Twist: A myogenic switch in Drosophila. Science 272: 1481-1484.

Baylies, M.K., A.M. Arias, and M. Bate. 1995. wingless is required for the formation of a subset of muscle founder cells during Drosophila embryogenesis. Development 121: 38293837.

Beer, J., G. Technau, and J. Campos Ortega. 1987. Lineage analysis of transplanted individual cells in embryos of Drosophila melanogaster. IV. Commitment and proliferative capabilities of mesodermal cells. Roux's Arch. Dev. Biol. 196: 222230.

Beiman, M., B.Z. Shilo, and T. Volk. 1996. Heartless, a Drosophila FGF receptor homolog, is essential for cell migration and establishment of several mesodermal lineages. Genes \& Dev. 10: 2993-3002.

Binari, R.C., B.E. Staveley, W.A. Johnson, R. Godavarti, R. Sasisekharan, and A.S. Manoukian. 1997. Genetic evidence that heparin-like glycosaminoglycans are involved in wingless signaling. Development 124: 2623-2632.

Bodmer, R. 1993. The gene tinman is required for specification of the heart and visceral muscles in Drosophila. Development 118: 719-729.

Brand, A.H. and N. Perrimon. 1993. Targeted gene expression as a means of altering cell fates and generating dominant phenotypes. Development 118: 401-415.

Buff, E., A. Carmena, S. Gisselbrecht, F. Jiménez, and A.M. Michelson. 1998. Signalling by the Drosophila epidermal growth factor receptor is required for the specification and diversification of embryonic muscle progenitors. Development 125: 2075-2086.

Carmena, A., M. Bate, and F. Jiménez. 1995. lethal of scute, a proneural gene, participates in the specification of muscle progenitors during Drosophila embryogenesis. Genes \& Dev. 9: 2373-2383.

Carmena, A., B. Murugasu-Oei, D. Menon, F. Jiménez, and W. Chia. 1998. inscuteable and numb mediate asymmetric muscle progenitor cell divisions during Drosophila myogen- esis. Genes \& Dev. 12: 304-315.

Clandinin, T., J. DeModena, and P. Sternberg. 1998. Inositol trisphosphate mediates a RAS-independent response to LET23 receptor tyrosine kinase activation in C. elegans. Cell 92: 523-533.

Corbin, V., A.M. Michelson, S.M. Abmayr, V. Neel, E. Alcamo, T. Maniatis, and M.W. Young. 1991. A role for the Drosophila neurogenic genes in mesoderm differentiation. Cell 67: 311-323.

Currie, P.D. and P.W. Ingham. 1998. The generation and interpretation of positional information within the vertebrate myotome. Mech. Dev. 73: 3-21.

Dunin Borkowski, O.M., N.H. Brown, and M. Bate. 1995. Anterior-posterior subdivision and the diversification of the mesoderm in Drosophila. Development 121: 4183-4193.

Edgar, B.A. and P.H. O'Farrell. 1989. Genetic control of cell division patterns in the Drosophila embryo. Cell 57: 177187.

Frasch, M. 1995. Induction of visceral and cardiac mesoderm by ectodermal Dpp in the early Drosophila embryo. Nature 374: 646-467.

Frasch, M., T. Hoey, C. Rushlow, H. Doyle, and M. Levine. 1987. Characterization and localization of the even-skipped protein of Drosophila. EMBO J. 6: 749-759.

Freeman, M. 1996. Reiterative use of the EGF receptor triggers differentiation of all cell types in the Drosophila eye. Cell 87: 651-660.

Gabay, L., R. Seger, and B.-Z. Shilo. 1997a. In situ activation pattern of Drosophila EGF receptor pathway during development. Science 277: 1103-1106.

- 1997b. MAP kinase in situ activation atlas during Drosophila embryogenesis. Development 124: 3535-3541.

Gisselbrecht, S., J.B. Skeath, C.Q. Doe, and A.M. Michelson. 1996. Heartless encodes a fibroblast growth factor receptor (DFR1/DFGF-R2) involved in the directional migration of early mesodermal cells in the Drosophila embryo. Genes \& Dev. 10: 3003-3017.

Golembo, M., E. Raz, and B.-Z. Shilo. 1996. The Drosophila embryonic midline is the site of Spitz processing, and induces activation of the EGF receptor in the ventral ectoderm. Development 122: 3363-3370.

Greenwald, I. and G.M. Rubin. 1992. Making a difference: The role of cell-cell interactions in establishing separate identities for equivalent cells. Cell 68: 271-281.

Greig, S. and M. Akam. 1993. Homeotic genes autonomously specify one aspect of pattern in the Drosophila mesoderm. Nature 362: 630-632.

Hartenstein, A.Y., A. Rugendorff, U. Tepass, and V. Hartenstein. 1992. The function of the neurogenic genes during epithelial development in the Drosophila embryo. Development 116: 1203-1220.

Jiang, J. and G. Struhl. 1996. Complementary and mutually exclusive activities of Decapentaplegic and Wingless organize axial patterning during Drosophila leg development. Cell 86: 401-409.

Katz, W.S., R.J. Hill, T.R. Clandinin, and P.W. Sternberg. 1995 Different levels of the C. elegans growth factor LIN-3 promote distinct vulval precursor fates. Cell 82: 297-307.

Kornfeld, K. 1997. Vulval development in Caenorhabditis elegans. Trends Genet. 13: 55-61.

Lawrence, P.A. 1992. The making of a fly. Blackwell Scientific Publications, Boston, MA.

Lawrence, P.A., R. Bodmer, and J.-P. Vincent. 1995. Segmental patterning of heart precursors in Drosophila. Development 121: 4303-4308.

Leptin, M. 1995. Drosophila gastrulation: From pattern forma- 
tion to morphogenesis. Annu. Rev. Cell Dev. Biol. 11: 189212.

Lüer, K., J. Urban, C. Klämbt, and G.M. Technau. 1997. Induction of identified mesodermal cells by CNS midline progenitors in Drosophila. Development 124: 2681-2690.

Marshall, C.J. 1995. Specificity of receptor tyrosine kinase signaling: Transient versus sustained extracellular signal-regulated kinase activation. Cell 80: 179-185.

Michelson, A.M., S. Gisselbrecht, Y. Zhou, K.-H. Baek, and E.M. Buff. 1998. Dual functions of the Heartless fibroblast growth factor receptor in development of the Drosophila embryonic mesoderm. Dev. Genet. 22: 212-229.

Park, M., X. Wu, K. Golden, J.D. Axelrod, and R. Bodmer. 1996. The Wingless signaling pathway is directly involved in Drosophila heart development. Dev. Biol. 177: 104-116.

Ranganayakulu, G., R.A. Schulz, and E.N. Olson. 1996. Wingless signaling induces nautilus expression in the ventral mesoderm of the Drosophila embryo. Dev. Biol. 176: 143-148.

Ray, R.P., K. Arora, C. Nüsslein-Volhard, and W.M. Gelbart. 1991. The control of cell fate along the dorsal-ventral axis of the Drosophila embryo. Development 113: 35-54.

Riechmann, V., U. Irion, R. Wilson, R. Grosskortenhaus, and M. Leptin. 1997. Control of cell fates and segmentation in the Drosophila mesoderm. Development 124: 2915-2922.

Riechmann, V., K.-P. Rehorn, R. Reuter, and M. Leptin. 1998. The genetic control of the distinction between fat body and gonadal mesoderm in Drosophila. Development 125: 713723.

Ruiz Gomez, M. and M. Bate. 1997. Segregation of myogenic lineages in Drosophila requires Numb. Development 124: 4857-4866.

Rushton, E., R. Drysdale, S.M. Abmayr, A.M. Michelson, and M. Bate. 1995. Mutations in a novel gene, myoblast city, provide evidence in support of the founder cell hypothesis for Drosophila muscle development. Development 121: 1979-1988.

Seger, R. and E.G. Krebs. 1995. The MAPK signaling cascade. FASEB J. 9: 726-735.

Shishido, E., N. Ono, T. Kojima, and K. Saigo. 1997. Requirements of DFR1/Heartless, a mesoderm-specific Drosophila FGF-receptor, for the formation of heart, visceral and somatic muscles, and ensheathing of longitudinal axon tracts in CNS. Development 124: 2119-2128.

Simpson, P. 1997. Notch signaling in development. Perspect. Dev. Neurobiol. 4: 297-304.

Staehling-Hampton, K., F.M. Hoffmann, M.K. Baylies, E. Rushton, and M. Bate. 1994. $d p p$ induces mesodermal gene expression in Drosophila. Nature 372: 783-786.

Stern, C. 1954. Two or three bristles? Am. Sci. 42: 213-247.

Tautz, D. and C. Pfeifle. 1989. A nonradioactive in situ hybridization method for the localization of specific RNAs in Drosophila embryos reveals a translational control of the segmentation gene hunchback. Chromosoma 98: 81-85.

van der Geer, P., T. Hunter, and R.A. Lindberg. 1994. Receptorprotein tyrosine kinases and their signal transduction pathways. Annu. Rev. Cell Biol. 10: 251-337.

Vervoort, M., C. Dambly-Chaudiere, and A. Ghysen. 1997. Cell fate determination in Drosophila. Curr. Opin. Neurobiol. 7: 21-28.

Wu, X., K. Golden, and R. Bodmer. 1995. Heart development in Drosophila requires the segment polarity gene wingless. Dev. Biol. 169: 619-628. 


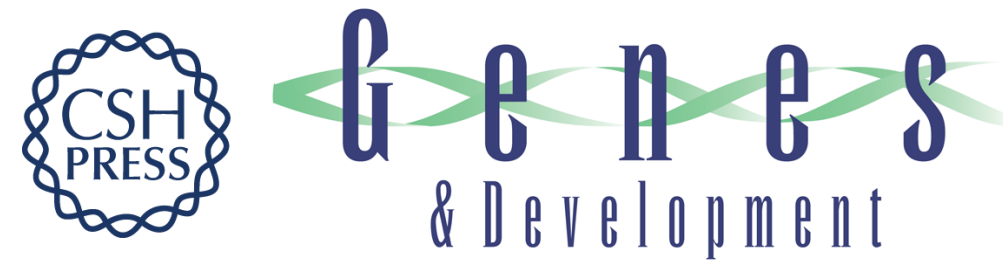

\section{Combinatorial signaling codes for the progressive determination of cell fates in the Drosophila embryonic mesoderm}

Ana Carmena, Stephen Gisselbrecht, Jacob Harrison, et al.

Genes Dev. 1998, 12:

Access the most recent version at doi:10.1101/gad.12.24.3910

References

This article cites 55 articles, 28 of which can be accessed free at:

http://genesdev.cshlp.org/content/12/24/3910.full.html\#ref-list-1

License

Email Alerting

Receive free email alerts when new articles cite this article - sign up in the box at the top

Service right corner of the article or click here.

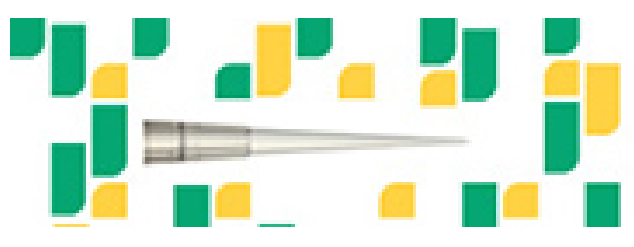

Focused on your science. 